\title{
Fecal Calprotectin and the Clinical Activity Index Are Both Useful to Monitor Medical Treatment in Patients with Ulcerative Colitis
}

\author{
Emanuel Burri · Christoph Beglinger • \\ Stefanie von Felten · Frank Serge Lehmann
}

Received: 18 September 2013/Accepted: 1 October 2014/Published online: 26 October 2014

(c) Springer Science+Business Media New York 2014

\begin{abstract}
Background Non-invasive monitoring of inflammatory bowel disease is an unmet clinical need as patients in clinical remission may have residual mucosal inflammation preceding clinical relapse.

Aims We aimed to assess the value of fecal calprotectin and standardized clinical activity scoring to monitor disease activity in ulcerative colitis under medical treatment. Methods Forty-one patients with ulcerative colitis were included in a prospective observational study. Medical treatment was guided by clinical judgement of treating physicians. Fecal calprotectin and the clinical activity index (CAI) were measured blinded to treating physicians every 2 months until the end of follow-up. Twenty-six patients received colonoscopy for clinical reason.

Results As defined by the CAI, patients were in clinical remission $(63.4 \%)$, having mild $(26.8 \%)$ or moderate
\end{abstract}

Electronic supplementary material The online version of this article (doi:10.1007/s10620-014-3383-0) contains supplementary material, which is available to authorized users.

\footnotetext{
E. Burri

Department of Gastroenterology, University Medical Clinic, Cantonal Hospital, Rheinstrasse 24, 4410 Liestal, Switzerland

e-mail: emanuel.burri@ksbl.ch

C. Beglinger $(\bowtie) \cdot$ F. S. Lehmann

Department of Gastroenterology and Hepatology, University

Hospital Basel, Petersgraben 4, 4031 Basel, Switzerland

e-mail: beglinger@tmr.ch

F. S. Lehmann

e-mail: fslehmann@hin.ch

S. von Felten

Clinical Trial Unit, University Hospital, Schanzenstrasse 55,

4031 Basel, Switzerland

e-mail: stefanie.vonfelten@usb.ch
}

$(11.2 \%)$ disease activity. Of those in clinical remission $(\mathrm{CAI} \leq 4), 86.4 \%$ showed residual endoscopic activity (Mayo Score $\geq 1$ ). Calprotectin levels were higher in endoscopically active disease $(779.0$ vs $331.5 \mu \mathrm{g} / \mathrm{g}$, $P=0.034)$ and calprotectin testing identified more patients with endoscopic disease activity $(86.4 \%)$ than the CAI (45.5\%, $P=0.034)$. Medical treatment was escalated in $90.2 \%$ during the study. Values of the CAI and calprotectin correlated with therapy escalation (OR 3.94 and 3.22 , respectively). Only for calprotectin, changes between two measurements were related to intensified medical treatment (OR 1.39).

Conclusion Fecal calprotectin was similarly useful to the CAI to monitor disease activity of ulcerative colitis during medical treatment but identified endoscopic disease activity far more reliably. Changes of calprotectin values between measurements might indicate clinical relapse earlier than the CAI.

Keywords Ulcerative colitis - Fecal calprotectin - Signs and symptoms, digestive $\cdot$ Drug therapy $\cdot$ Colonoscopy

\section{Background}

Inflammatory bowel disease (IBD) is a chronic disorder that is characterized by a remitting and relapsing clinical course [1]. Symptoms are often unspecific and may vary with the disease course making it difficult to clinically assess disease activity. In clinical trials, a variety of clinical indices have been used to measure disease activity and response to treatment [2, 3]. However, these indices strongly rely on subjective patient symptoms and often depend on nonspecific serum markers of systemic inflammation. Improvement of clinical symptoms would usually 
indicate decrease of disease activity and accordingly guide medical treatment in daily practice. However, this approach has disadvantages: first, symptoms of intestinal inflammation are often nonspecific and may prove unreliable when evaluating disease activity. Second, IBD patients with clinically quiescent disease may have residual mucosal inflammation [4] and remission of symptoms may not indicate remission of IBD.

Residual inflammation in quiescent IBD may constitute subclinical disease activity and represent an early stage of relapse. The assessment of treatment response and monitoring of disease activity are therefore important issues and present an unmet clinical need, both in ulcerative colitis (UC) and Crohn's disease (CD). To date, endoscopic assessment of mucosal inflammation is still the gold standard to assess disease activity in IBD $[5,6]$ but it is invasive, costly, and uncomfortable for patients. Clearly, the need for a reliable, noninvasive test to monitor disease activity and predict relapse of IBD is paramount.

In recent years, measurement of fecal calprotectin (FC) has emerged as a noninvasive biomarker to detect intestinal inflammation [7-9]. Calprotectin is a calcium binding protein mainly found in neutrophils [10] and when measured in feces, correlates with neutrophilic migration to the intestinal mucosa [11]. In UC, levels of FC have been shown to correlate well with endoscopic and also histological parameters of disease activity [12-15]. Current data suggest that elevated FC may predict clinical relapse in patients with quiescent disease [16-19], presumably by detecting residual inflammation in patients with clinical remission [20].

Data on serial measurement of FC are scarce. FC has been assessed at different time points in adult and pediatric IBD patients and correlated to serum parameters, clinical activity scores and endoscopy [21-24]. Serial measurement of FC might detect increased disease activity earlier, i.e., before any clinical symptoms have occurred, and therefore help to guide UC therapy. Data on the clinical benefit and improved long-term outcome of UC by calprotectin-guided medical treatment are not available so far.

The aim of this study was to compare the value of serial FC measurements and standardized clinical scoring in the assessment of UC disease activity during medical treatment guided by clinical judgement and to investigate if FC would indicate the need for treatment escalation earlier.

\section{Methods}

Setting and Participants

In this observational study, we prospectively investigated patients with UC referred for medical treatment to the
Department of Gastroenterology of the University Hospital Basel in Switzerland from August 2010 to January 2012. The diagnosis of UC had been established prior to referral according to international guidelines including endoscopy and histology or was made before study inclusion [5]. Exclusion criteria were incomplete diagnostic work-up, age younger than 18 years, a medical history of primary immunodeficiency, underlying chronic or active malignant disease and current infection. Abdominal surgery was not allowed within 1 month prior to study entry. Patients were followed until end of study. The study was carried out according to the Principles of the Declaration of Helsinki, and the local ethical committee approved the study protocol. All patients gave written informed consent before participating in any protocol-specific procedures.

\section{Endpoint}

The correlation of serial FC measurement and clinical activity scoring using the modified colitis activity index (CAI) by Rachmilewitz to assess disease activity was the primary endpoint of the study. As a secondary endpoint, we investigated the predictive value of these measurements for the need to intensify medical treatment. FC values equal to or greater than $50 \mu \mathrm{g} / \mathrm{g}$ were considered a positive test result.

\section{Diagnosis of Ulcerative Colitis}

The diagnosis of UC was adjudicated on the basis of all available medical records pertaining to the individual patient (clinical data, laboratory values, radiology report, endoscopy report, histology report) according to current guidelines [5]. The extent of UC was based on the Montreal Classification [25]. Patients had either ulcerative proctitis, left sided UC or extensive disease.

\section{Endoscopic Assessment}

Endoscopic assessment of disease activity was done using the Mayo ulcerative colitis endoscopic score and graded to be normal or inactive disease (score $=0$ ), mild disease (erythema, decreased vascular pattern, friability; score $=1$ ), moderate disease (marked erythema, absent vascular pattern, friability, erosions; score $=2$ ) and severe disease (spontaneous bleeding, ulceration; score $=3$ ) [26] The assessment was done blinded to FC values and the results of clinical activity scoring.

\section{Clinical Activity Index}

Clinical activity was assessed using the CAI (Table A, Supplements) at study entry and consecutively every 2 months until end of follow up [27]. The index combines 
objective measurements (number of stools in 1 week, temperature, extraintestinal manifestations) with subjective findings (blood in stool, abdominal pain/cramps, global assessment of symptomatic state). The total index score ranges from 0 to 25 . Degrees of clinical activity were defined as follows: inactive (remission) $0-4$, mild activity $5-10$, moderate activity $11-17$, and high activity $\geq 18$ points [12]. The evaluation of clinical activity by the CAI was performed by a study nurse who was not involved in clinical care. All patients were instructed to contact the physician if there was any change in clinical symptoms. A clinical relapse was defined as an increase of the CAI to a score $>4$ points in patients with clinical remission.

\section{Measurement of Fecal Calprotectin}

FC was measured at study entry and consecutively every 2 months (together with the CAI). Stool samples were collected at home and sent directly to the laboratory. The laboratory personnel carrying out the analysis was blinded to the clinical history of the patients. All fecal samples were processed within $72 \mathrm{~h}$ after collection.

FC was determined using a commercially available enzyme-linked immunosorbent assay (Bühlmann Laboratories, Schönenbuch, Switzerland) that measures quantitative calprotectin as previously described [28]. Briefly, aliquots of approximately $100 \mathrm{mg}$ feces were homogenized and centrifuged $(5 \mathrm{~min}$ at $2,000 \mathrm{~g}$ ) and $100 \mu \mathrm{l}$ of the diluted supernatant (1:50 with incubation buffer) were incubated at room temperature onto a microtiter plate coated with a monoclonal capture antibody highly specific to the calprotectin heterodimeric and polymeric complexes. After incubation, washing and adding a detection antibody coupled to horseradish peroxidase, tetramethylbenzidine was added, which was then followed by a stop solution. The absorption was determined at an optical density of $450 \mathrm{~nm}$. A positive value was defined as equal or greater than $50 \mu \mathrm{g} / \mathrm{g}$ as stated by the manufacturer.

\section{Medical Treatment}

The decision to start or change medical treatment was made according to clinical judgment, including patient's symptoms. Treating physicians were blinded to results of individual FC values and to scores of the CAI at the time of the patients visit. The CAI was calculated from questionnaires assessed by a study nurse independent of patient care. Intensification of medical therapy was defined as follows: (a) new prescription of any IBD medication in untreated patients, (b) increase of dosage of any previously established medication, and (c) change of medication to glucocorticoids, anti TNF-alpha inhibitors (infliximab) or immunosuppressants (azathioprine, mycophenolate).
Statistical Analysis

The statistical analysis was performed using the MedCalc (version 12.3.0, MedCalc Software, Belgium) and R (version 2.15.1, R Foundation for Statistical Computing, Austria) software packages. All hypothesis testing was twotailed, using a significance level of 0.05 . The relationship between FC values and the CAI score was assessed using a linear mixed-effects model allowing random intercepts for individuals (repeated FC measurements and clinical score taking). Values were log-transformed (after addition of one for values of zero) if necessary. The relationships between FC values and therapy intensification and between the CAI score and therapy intensification were analyzed with the generalized estimating equation (GEE). Thereby, we fitted models using both absolute (log-transformed) and changes in FC (\% change relative to the previous measurement) and CAI scores (absolute difference between subsequent measurements). The number of subjects included in the models was different, if absolute values $(N=41)$ or changes between measurements $(N=40)$ of FC and the CAI were used. Receiver operating characteristics (ROC) analyses including calculations of the area under the curve (AUC) were carried out to determine the capability of FC (absolute values and relative change), the CAI scores (absolute score and change) and a combination of both (calculated by logistic regression analysis) to predict the need for therapy intensification within the next 2 months. As this was an exploratory study, no formal power calculations were performed.

\section{Results}

\section{Patient Characteristics}

A total of 41 patients (15 male, 26 female, 22-83 years of age) with UC were recruited for the study. One female patient was excluded from the final analysis, as only a single fecal sample was provided. Baseline characteristics are shown in Table 1. Mean disease duration was 12.3 years (standard deviation 9.2, range 0-30). At the time of inclusion in the study, 11 patients $(26.8 \%)$ were treated with 5-ASA alone, nine patients $(22 \%)$ in combination with either topical or systemic glucocorticoids, eight patients (19.5\%) were taking immunosuppressants and seven patients $(17.1 \%)$ received anti TNFalpha therapy. Five patients had no therapy. Mean time of follow-up was $9.3 \pm 0.6$ months (range 2-14) and was $\geq 12$ months in 19 patients $(46.3 \%)$. During the study period, no patient was hospitalized for severe UC or underwent surgery for UC-related complications. No patient died. 
Table 1 Baseline characteristics

\begin{tabular}{ll}
\hline Variable & Value \\
\hline Age, mean (SD) & $48.2(22-83)$ \\
Female gender, $n(\%)$ & $26(63.4)$ \\
Disease duration, mean (SD) & $12.3(9.2)$ \\
Disease extent, $n(\%)$ & \\
Extensive UC/pancolitis & $11(26.8)$ \\
Left-sided UC/distal UC & $24(58.5)$ \\
Ulcerative proctitis & $6(14.6)$ \\
Drugs at study entry, $n(\%)$ & \\
Topical/systemic 5-ASA & $20(48.8)$ \\
Topical/systemic steroids & $14(34.1)$ \\
Azathioprine & $5(12.2)$ \\
Mycophenolate & $2(4.9)$ \\
Cyclosporin & $1(2.4)$ \\
Infliximab & $7(17.1)$ \\
No medication & $5(12.2)$ \\
\hline
\end{tabular}

Data are presented as mean (standard deviation, SD) and number of patients $(\%)$

5-ASA 5-aminosalicylic acids

\section{Clinical Activity Index}

At study entry, CAI values ranged from 0 to 13 points [mean 4.2, standard error (SE) \pm 0.6 ]. As defined by the CAI, 26 patients $(63.4 \%)$ were classified to be in clinical remission at study entry, 11 patients $(26.8 \%)$ to have mildly active disease and four patients $(9.8 \%)$ to have moderate disease activity. No patient had highly active disease. FC values were higher in patients with clinically active disease than in clinical remission $(779.0 \mu \mathrm{g} / \mathrm{g}$, interquartile range (IQR) $345.0-1,752.8$ vs $331.5 \mu \mathrm{g} / \mathrm{g}$, IQR 102.0-752.0, $P=0.034$ ).

\section{Endoscopic Disease Activity}

Ileo-colonoscopy was performed in 26 patients $(63.4 \%)$ for clinical reason during the study period, either at study inclusion $(N=20)$ or within 3 months thereafter $(N=6)$. FC levels were lower in patients with Mayo UC endoscopic score $1(N=5,79.0 \mu \mathrm{g} / \mathrm{g}$, IQR $31.5-167.0)$ than with Mayo UC endoscopic score $2(N=6,329.0 \mu \mathrm{g} / \mathrm{g}$, IQR 234.0-438.0, $P=0.038)$ and score $3(N=11,1,890.0 \mu \mathrm{g} /$ g, IQR 640.5-3,671.8, $P=0.011$; Fig. 1). Patients with endoscopic remission $(N=4)$ had similar median calprotectin values than patients with mildly active disease (92.5 $\mu \mathrm{g} / \mathrm{g}$, IQR 53.5-139.5 vs 79.0 $\mu \mathrm{g} / \mathrm{g}$, IQR 31.5-167.5, $P=0.713)$. The correlation between the Mayo UC endoscopic score and FC was 0.754 (CI 0.52-0.88, $P<0.001$ ).

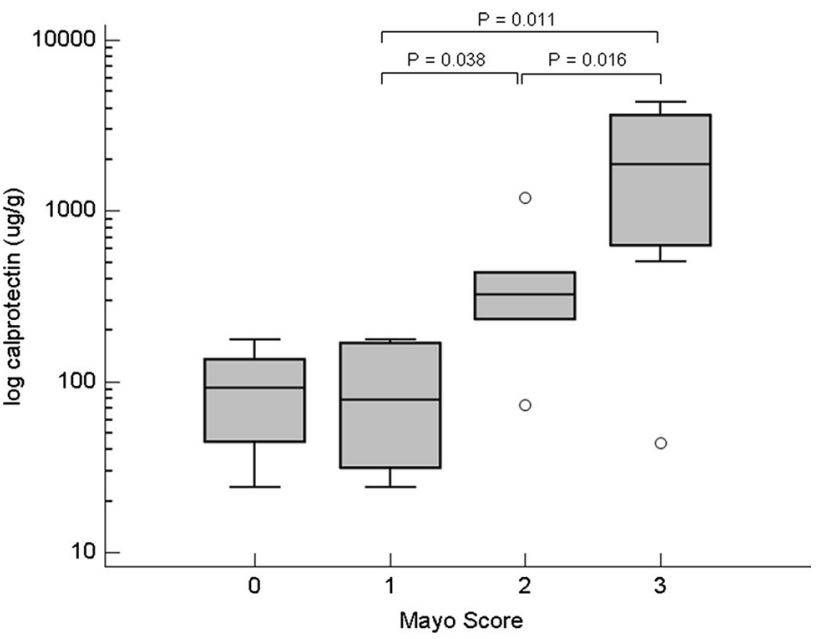

Fig. 1 Fecal calprotectin and endoscopic disease activity. Fecal calprotectin values of patients according to the Mayo ulcerative colitis endoscopic activity score: normal or inactive disease $($ score $=0$ ), mild disease (erythema, decreased vascular pattern, friability; score $=1$ ), moderate disease (marked erythema, absent vascular pattern, friability, erosions; score $=2$ ) and severe disease (spontaneous bleeding, ulceration, score $=3$ )

\section{Treatment Escalation}

Medical treatment was escalated for clinical reason at any point during the study period in 37 patients $(90.2 \%$; in 31 patients at the initial visit). Interestingly, when assessed with the CAI, only 18 patients $(48.6 \%)$ had clinically active disease (mean $4.8 \pm 0.6$ ) at the time of escalation. Additionally, 19 of 22 patients $(86.4 \%)$ with UC supposedly in clinical remission according the CAI ( $\leq 4$ points) had active endoscopic disease (Mayo UC endoscopic subscore $\geq 1$ ), suggesting a systematic underestimation of endoscopic disease activity by the CAI.

In addition, FC values were higher in patients in need for intensified medical treatment [median $576 \mu \mathrm{g} / \mathrm{g}$ (IQR 289-1,398) vs $69 \mu \mathrm{g} / \mathrm{g}$ (IQR 29-354), $P=0.037]$ and, except for one patient, all had positive FC testing $(>50 \mu \mathrm{g} /$ g) at treatment escalation. In our study group, FC in patients with endoscopic remission $(N=4)$ was $92.5 \mu \mathrm{g} / \mathrm{g}$ (see above).

\section{Correlation of CAI and Calprotectin During Treatment}

Two hundred thirty-three questionnaires (response rate $96.6 \%)$ to assess CAI and 223 fecal samples were collected $(95.7 \%)$ for analysis. Under treatment, the CAI decreased to $1.2 \pm 0.2$ and $95.1 \%$ of all patients had $\mathrm{CAI} \leq 4$ at the end of follow-up $(P<0.001$ against basal for both). Similarly, calprotectin values decreased to $93.5 \mu \mathrm{g} / \mathrm{g}, \mathrm{IQR} 33.0-170.0$ ( $P=0.002$ against basal), and 


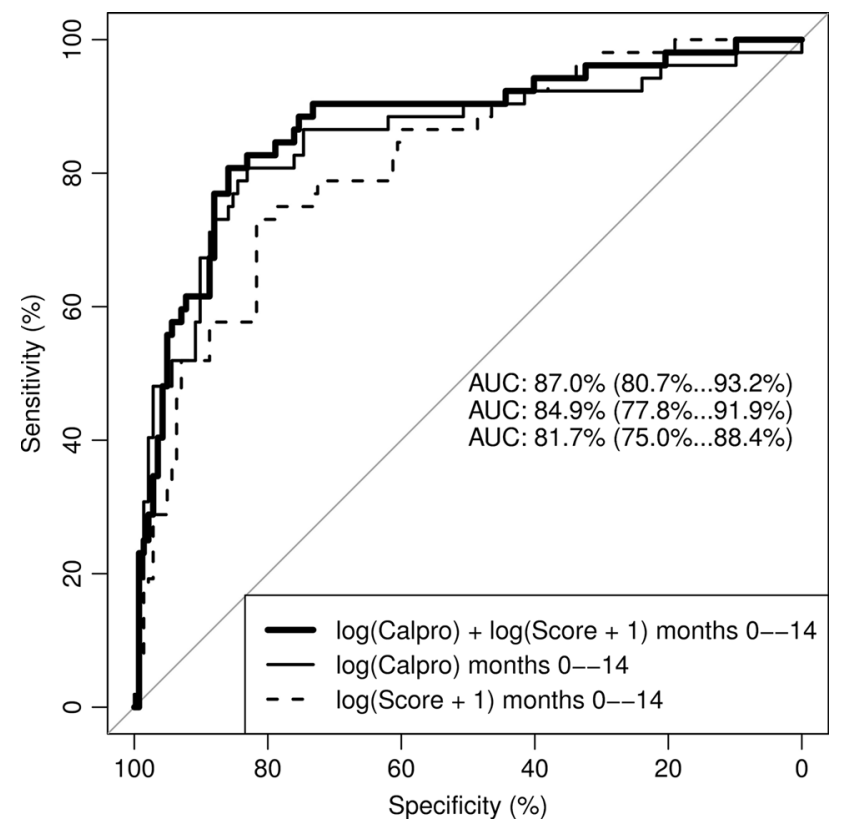

Fig. 2 Receiver operating characteristics curve. Receiver operating characteristics analysis showing a larger area under the curve (AUC) for fecal calporotectin values, log (Calpro), than for clinical activity scores, $\log ($ Score +1$) ; P=0.029$. The combination of both parameters, $\log ($ Calpro $)+\log ($ Score +1$)$, did not increase the predictive capability $(P=0.153$ against fecal calprotectin alone)

normalized $(<50 \mu \mathrm{g} / \mathrm{g})$ in $41.5 \% \quad(P=0.004$ against basal). The linear mixed-effects model estimated a positive correlation between the two parameters (estimate 0.26, $95 \%$ CI $0.18-0.34, P<0.001)$ during serial measurement.

\section{CAI and Calprotectin Levels to Guide Therapy}

Values of the CAI correlated with the need to intensify medical treatment (odds ratio (OR) 3.94, $95 \%$ CI $2.29-6.78, P<0.001)$. Evaluating the predictive ability of the CAI treatment escalation, ROC analysis found an AUC of 0.817 (Fig. 2). Calprotectin values also correlated with the need for intensified UC therapy (OR 3.22, $95 \%$ CI 2.07-5.02, $P<0.001)$ and predicted treatment escalation (AUC of $0.849, P=0.029$ against CAI). In addition, only changes of fecal calprotectin, e.g. an increase or a decrease, between measurements every 2 months were related to intensified medical treatment (OR 1.39, $95 \%$ CI $1.06-1.83, P=0.020)$. This was not true for the CAI (OR $1.16,95 \%$ CI $0.93-1.44, P=0.19)$, suggesting better prediction of clinical relapse by calprotectin testing.

\section{Discussion}

This prospective observational study examined the use of standardized clinical activity scoring and serial measurement of FC to monitor disease activity and predict therapy intensification in patients with UC. We provide the following findings. First, the CAI and FC values correlated during medical treatment. Second, both the CAI and FC values predicted the need for therapy intensification based on clinical judgment. FC suggested doing so earlier than the CAI. Third, FC was superior to the CAI in identifying endoscopically active disease. Fourth, FC values correlated well with the degree of mucosal inflammation. These findings are of clinical importance as they provide evidence on the value of serial FC measurements in the management of patients with active UC, especially to identify clinically quiescent disease.

Endoscopy is still the gold standard to characterize and grade mucosal inflammation. However, endoscopy is an invasive and costly procedure that cannot be recommended to closely monitor disease activity. In the last decade, FC has emerged as surrogate marker of intestinal inflammation and has proven useful in the management of IBD [29]. In the present study, FC reliably identified disease activity in the subset of UC patients that received endoscopy and correlated well with the degree of mucosal inflammation. These results confirm prior data by our group which also found FC to correlate better with endoscopic activity ( $R=0.834)$ compared to the CAI $(R=0.672)$ and/or to blood testing (CRP, leucocytes) [12]. In another study, the sensitivity and specificity of FC to identify endoscopic activity (modified Baron score $\geq 2$ ) were 91 and $90 \%$, respectively (cut-off $57 \mu \mathrm{g} / \mathrm{g})$ [15]. Again, FC $(R=0.821)$ performed better than standardized clinical scoring (by the Lichtiger index, $R=0.682$ ) and blood tests.

Unfortunately, the definition of mucosal healing in most endoscopic activity scores, including the Mayo endoscopic subscore, is not clearly defined and not well established. Accordingly, there is no consensus on FC levels that would indicate mucosal healing in patients with established IBD. Research on the role of FC as surrogate marker is still ongoing.

The evaluation of treatment response in IBD has traditionally been based on clinical symptoms, activity scores and serum markers of inflammation but only $70 \%$ also have endoscopic remission [30]. Ongoing low-grade inflammation might indicate an increased risk of clinical relapse. FC has been investigated as predictor of IBD relapse [16-19, 31] and pooled sensitivity and specificity of FC (cut-off $50-340 \mu \mathrm{g} / \mathrm{g}$ ) in clinical remission was reported to be 78 and $73 \%$, respectively [32]. However, most of these studies were limited by their design using a single test rather than serial FC measurements to predict the clinical course during therapy and follow-up. Data on sequential FC testing is limited [21-24].

In our study, medical treatment for UC was escalated in 37 patients $(90.2 \%)$ at any point during follow-up and both the CAI and FC values decreased under appropriate 
therapy. To predict the need for treatment escalation, both the CAI and FC values were useful but, additionally, we found that changes in FC concentrations between two measurements, e.g. increase or decrease, were also predictive for intensified UC therapy. This could suggest that FC might indicate the need for therapy escalation earlier than the CAI. These results are further supported by De Vos et al. [33]. They found that in UC patients during clinical remission, loss of response to infliximab during maintenance therapy corresponded with a significant increase in FC levels as much as 3 months before the appearance of clinical symptoms.

The ultimate goal of using the prognostic value of FC to identify (endoscopically) active IBD before a clinical relapse has occurred and changing the disease course by escalating medical treatment prior to symptoms occurrence still awaits confirmation by large, prospective trials.

Some limitations of this study need to be addressed. First, this was an exploratory study that investigated a limited number of UC patients. The study was neither designed nor powered to investigate the clinical IBD course as a result of earlier treatment escalation by calprotectin measurements. Clearly, future randomized controlled trials will have to further explore the prognostic value of fecal calprotectin to identify (endoscopically) active IBD before a clinical relapse has occurred and by escalating medical treatment prior to symptom occurrence, reaching the ultimate goal of changing the disease course. Second, treating physicians might have introduced a systemic error by simply mirroring the CAI trough their clinically-based treatment decisions. Although some aspects of the CAI should be part of any clinical assessment in UC patients, we tried to minimize this bias. The CAI was independently assessed by someone not involved in clinical care and treating physicians were blinded to the results. Third, there was no supervision of treatment decisions and clinical care was not standardized. However, all participating physicians adhered to ECCO recommendations to guide medical therapy and a specific definition of treatment escalation was given in the protocol (see "Methods" section). Although some uncertainty may remain whether patients were all treated appropriately, the goal of this observational study was to assess the value of FC in monitoring clinical disease activity and any inappropriate treatment (e.g. incorrect escalation of therapy) would have been reflected in the clinical course, and accordingly in the CAI and FC values. Fourth, we did not systematically assess endoscopic disease activity. However, in the subset of patients that received endoscopy, we confirmed the positive correlation and the diagnostic superiority of FC to identify endoscopically active disease $[21,23,34]$.
In conclusion, our results show that $\mathrm{FC}$ is a reliable tool to identify endoscopically active UC and is helpful to monitor disease activity and decide on treatment escalation. Clinical assessment with the CAI correlated with FC values during therapy but did not identify a substantial number of patients with endoscopic disease activity. Whether a calprotectinguided treatment strategy would be advantageous to decrease relapse rates and increase the rate of endoscopically quiescent disease compared to a therapy based on clinical symptoms and activity scores needs to be further investigated.

Acknowledgments Viollier AG (Basel, Switzerland) performed all measurements of FC. Researchers were independent of funding. We are indebted to the patients who participated in the study for their most valuable efforts.

Conflict of interest All authors report no conflict of interest.

\section{References}

1. Vatn MH. Natural history and complications of IBD. Curr Gastroenterol Rep. 2009;11:481-487.

2. Vilela EG, Torres HO, Martins FP. Evaluation of inflammatory activity in Crohn's disease and ulcerative colitis. World J Gastroenterol. 2012;18:872-881.

3. Sostegni R, Daperno M, Scaglione N, Lavagna A, Rocca R, Pera A. Review article: Crohn's disease: monitoring disease activity. Aliment Pharmacol Ther. 2003;17:11-17.

4. Saverymuttu SH. Clinical remission in Crohn's diseaseassessment using faecal 111In granulocyte excretion. Digestion. 1986;33:74-79.

5. Dignass A, Lindsay JO, Sturm A, et al. Second European evidence-based consensus on the diagnosis and management of ulcerative colitis part 2: current management. J Crohns Colitis. 2012;6:991-1030.

6. Dignass A, Van Assche G, Lindsay JO, et al. The second European evidence-based consensus on the diagnosis and management of Crohn's disease: current management. J Crohns Colitis. 2010;4:28-62.

7. Tibble JA, Sigthorsson G, Foster R, Forgacs I, Bjarnason I. Use of surrogate markers of inflammation and Rome criteria to distinguish organic from nonorganic intestinal disease. Gastroenterology. 2002;123:450-460.

8. Langhorst J, Elsenbruch S, Koelzer J, Rueffer A, Michalsen A, Dobos GJ. Noninvasive markers in the assessment of intestinal inflammation in inflammatory bowel diseases: performance of fecal lactoferrin, calprotectin, and PMN-elastase, CRP, and clinical indices. Am J Gastroenterol. 2008;103:162-169.

9. van Rheenen PF, Van de Vijver E, Fidler V. Faecal calprotectin for screening of patients with suspected inflammatory bowel disease: diagnostic meta-analysis. BMJ. 2010;341:c3369.

10. Foell D, Frosch M, Sorg C, Roth J. Phagocyte-specific calciumbinding S100 proteins as clinical laboratory markers of inflammation. Clin Chim Acta. 2004;344:37-51.

11. Roseth AG, Schmidt PN, Fagerhol MK. Correlation between faecal excretion of indium-111-labelled granulocytes and calprotectin, a granulocyte marker protein, in patients with inflammatory bowel disease. Scand J Gastroenterol. 1999;34:50-54.

12. Schoepfer AM, Beglinger C, Straumann A, Trummler M, Renzulli P, Seibold F. Ulcerative colitis: correlation of the 
Rachmilewitz endoscopic activity index with fecal calprotectin, clinical activity, C-reactive protein, and blood leukocytes. Inflamm Bowel Dis. 2009;15:1851-1858.

13. Ricanek P, Brackmann S, Perminow G, et al. Evaluation of disease activity in IBD at the time of diagnosis by the use of clinical, biochemical, and fecal markers. Scand $J$ Gastroenterol. 2011;46:1081-1091.

14. D'Haens G, Ferrante M, Vermeire S, et al. Fecal calprotectin is a surrogate marker for endoscopic lesions in inflammatory bowel disease. Inflamm Bowel Dis. 2012;18:2218-2224.

15. Schoepfer AM, Beglinger C, Straumann A, et al. Fecal calprotectin more accurately reflects endoscopic activity of ulcerative colitis than the Lichtiger Index, C-reactive protein, platelets, hemoglobin, and blood leukocytes. Inflamm Bowel Dis. 2013;19:332-341.

16. Tibble JA, Sigthorsson G, Bridger S, Fagerhol MK, Bjarnason I. Surrogate markers of intestinal inflammation are predictive of relapse in patients with inflammatory bowel disease. Gastroenterology. 2000;119:15-22.

17. Costa F, Mumolo MG, Ceccarelli L, et al. Calprotectin is a stronger predictive marker of relapse in ulcerative colitis than in Crohn's disease. Gut. 2005;54:364-368.

18. Gisbert JP, Bermejo F, Perez-Calle JL, et al. Fecal calprotectin and lactoferrin for the prediction of inflammatory bowel disease relapse. Inflamm Bowel Dis. 2009;15:1190-1198.

19. D'Incà R, Dal Pont E, Di Leo V, et al. Can calprotectin predict relapse risk in inflammatory bowel disease? Am J Gastroenterol. 2008;103:2007-2014.

20. Sipponen T, Kolho KL. Faecal calprotectin in children with clinically quiescent inflammatory bowel disease. Scand J Gastroenterol. 2010;45:872-877.

21. De Vos M, Dewit O, D’Haens G, et al. Fast and sharp decrease in calprotectin predicts remission by infliximab in anti-TNF naive patients with ulcerative colitis. J Crohns Colitis. 2012;6:557-562.

22. Gerasimidis K, Nikolaou CK, Edwards CA, McGrogan P. Serial fecal calprotectin changes in children with Crohn's disease on treatment with exclusive enteral nutrition: associations with disease activity, treatment response, and prediction of a clinical relapse. J Clin Gastroenterol. 2011;45:234-239.

23. Sipponen T, Savilahti E, Karkkainen P, et al. Fecal calprotectin, lactoferrin, and endoscopic disease activity in monitoring anti-
TNF-alpha therapy for Crohn's disease. Inflamm Bowel Dis. 2008;14:1392-1398.

24. Wagner M, Peterson CGB, Ridefelt P, Sangfelt P, Carlson M. Fecal markers of inflammation used as surrogate markers for treatment outcome in relapsing inflammatory bowel disease. World J Gastroenterol. 2008;14:5584-5589; discussion 5588.

25. Silverberg MS, Satsangi J, Ahmad T, et al. Toward an integrated clinical, molecular and serological classification of inflammatory bowel disease: report of a working party of the 2005 Montreal World Congress of Gastroenterology. Can J Gastroenterol. 2005; 19:5-36.

26. Schroeder KW, Tremaine WJ, Ilstrup DM. Coated oral 5-aminosalicylic acid therapy for mildly to moderately active ulcerative colitis. A randomized study. $N$ Engl $J$ Med. 1987:317:1625-1629.

27. Rachmilewitz D. Coated mesalazine (5-aminosalicylic acid) versus sulphasalazine in the treatment of active ulcerative colitis: a randomised trial. BMJ. 1989;298:82-86.

28. Manz M, Burri E, Rothen C, et al. Value of fecal calprotectin in the evaluation of patients with abdominal discomfort: an observational study. BMC Gastroenterol. 2012;12:5.

29. Burri E, Beglinger C. Faecal calprotectin-a useful tool in the management of inflammatory bowel disease. Swiss Med Wkly. 2012;142:w13557.

30. Modigliani R. Endoscopic management of inflammatory bowel disease. Am J Gastroenterol. 1994;89:S53-S65.

31. Garcia-Sanchez V, Iglesias-Flores E, Gonzalez R, et al. Does fecal calprotectin predict relapse in patients with Crohn's disease and ulcerative colitis? J Crohns Colitis. 2010;4:144-152.

32. Mao R, Xiao YL, Gao X, et al. Fecal calprotectin in predicting relapse of inflammatory bowel diseases: a meta-analysis of prospective studies. Inflamm Bowel Dis. 2012;18:1894-1899.

33. Vos MD, Louis EJ, Jahnsen J, et al. Consecutive fecal calprotectin measurements to predict relapse in patients with ulcerative colitis receiving infliximab maintenance therapy. Inflamm Bowel Dis. 2013;19:2111-2117.

34. Sipponen T, Bjorkesten CG, Farkkila M, Nuutinen H, Savilahti E, Kolho KL. Faecal calprotectin and lactoferrin are reliable surrogate markers of endoscopic response during Crohn's disease treatment. Scand J Gastroenterol. 2010;45:325-331. 